\title{
3D Tracking-Free Approach for Obtaining 3D Super-Resolution Information in Rotationally Symmetric Bio-Structures
}

\section{Andrew Ruba*, Wangxi Luo, Joseph Kelich, Mark Tingey, and Weidong Yang*} Affiliation: Department of Biology, Temple University, 1900 N. 12 $2^{\text {th }}$ St., Philadelphia, Pennsylvania, USA

* Corresponding authors: Weidong Yang, Email: weidong.yang@temple.edu; Tel: 215204-2312. Andrew Ruba, Email: andrew.ruba@temple.edu; Tel: 215-204-2243 


\section{Calculating the regions of the area matrix}

In the $2 \mathrm{D}$-to-3D density transformation process, we use an area matrix to reflect the contribution of each ring to the $2 \mathrm{D}$ distribution. As shown below, we define $\mathrm{i}$ as the radial number and $\mathrm{j}$ as the axial number. The density of single molecule locations in the same radius $i$ is supposed to be uniform given the radial symmetry and the density is labeled as $\rho_{i}$ here. The cross-section of radial number $\mathrm{i}$ and axial number $\mathrm{j}$ is defined as $\mathrm{A}_{(\mathrm{i}, \mathrm{j})}$. In the following equations, $\Delta r$ is the bin size, $\rho_{i}$ is the density of molecules in the $i$ th ring, $h$ is the half of illumination depth and $\rho$ is the constant background density outside the region of interest.
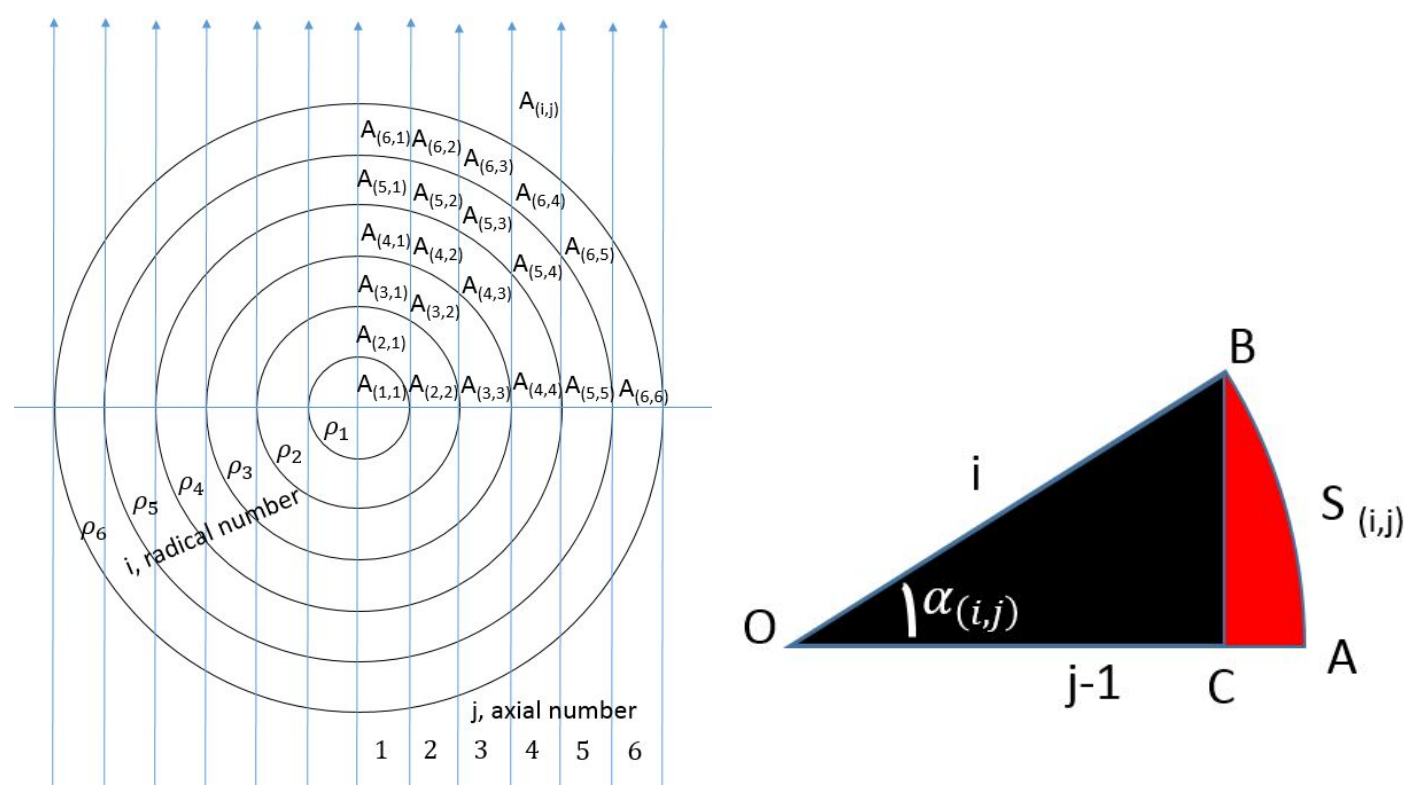

These above graphics represent the area matrix nomeclature and labeling of the are matrix section for calculating the red shaded region. 
To determine the area of each sub-region $\left(\mathrm{A}_{(\mathrm{i}, \mathrm{j})}\right)$, we always start to calculate the area of the fan-shape area at $j=i$, in which:

$\cos \alpha_{(i, j)}=\frac{j-1}{i}, \quad \sin \alpha_{(i, j)}=\frac{\sqrt{i^{2}-(j-1)^{2}}}{i}, \quad \alpha_{(i, j)}=\cos ^{-1} \frac{j-1}{i}$

The red-shaded area is defined as $\mathrm{S}_{(\mathrm{i}, \mathrm{j})}$

$\mathrm{S}_{(\mathrm{i}, \mathrm{j})}=\mathrm{S}_{\mathrm{ABC}}=\mathrm{S}_{\mathrm{AOB}}-\mathrm{S}_{\mathrm{BOC}}$

$$
S_{(i, j)}=\frac{1}{2} \alpha_{(i, j)} i^{2} \Delta \mathrm{r}^{2}-\frac{1}{2} \sin \alpha_{(i, j)} \cos \alpha_{(i, j)} i^{2} \Delta \mathrm{r}^{2}
$$

$S_{(i, j)}=\frac{1}{2}\left[\cos ^{-1}\left(\frac{j-1}{i}\right)\right] i^{2} \Delta \mathrm{r}^{2}-\frac{1}{2}\left[\frac{\sqrt{i^{2}-(j-1)^{2}}}{i}\right]\left(\frac{j-1}{i}\right) i^{2} \Delta \mathrm{r}^{2}$ 

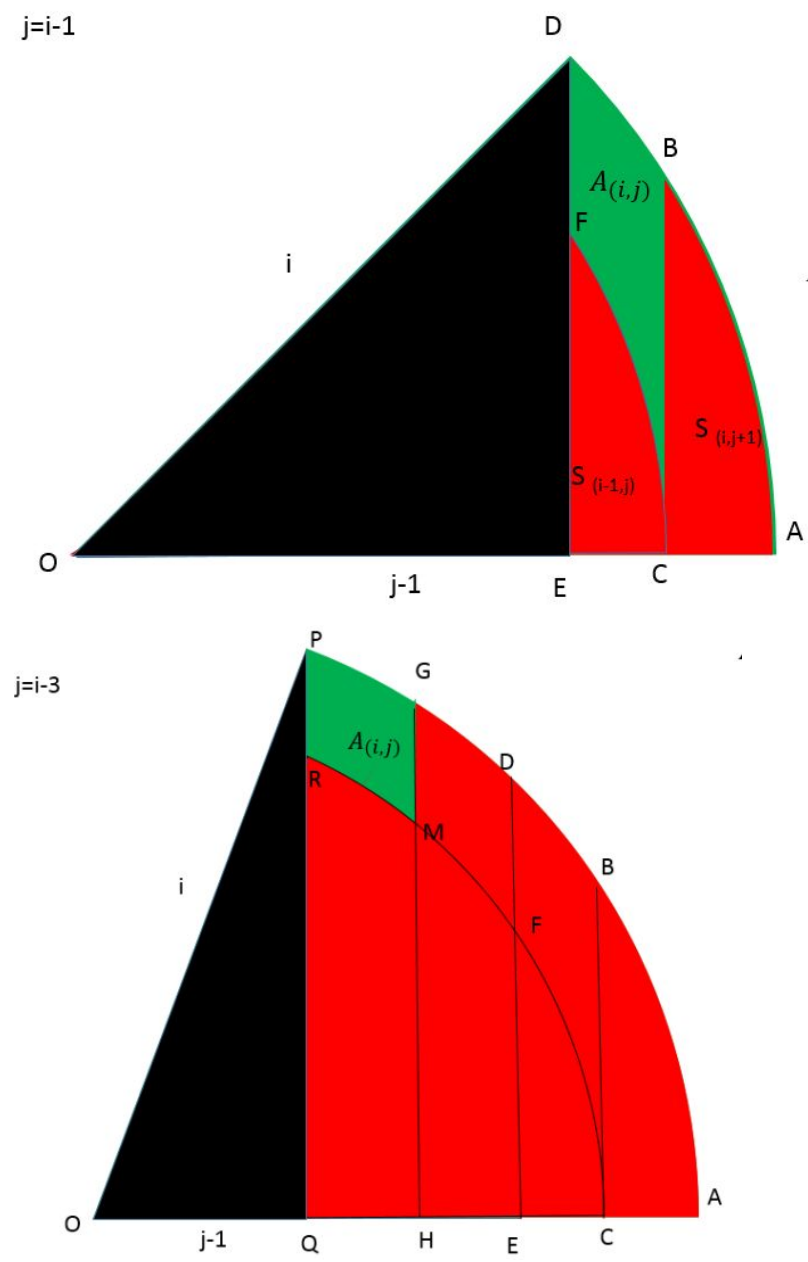

When $j \neq i$ and $i>j$, we need to calculate the area of the green-shaded region as follows:

$$
\begin{aligned}
& \mathrm{S}_{\mathrm{CBDF}}=\mathrm{S}_{\mathrm{ADE}}-\mathrm{S}_{\mathrm{CFE}}-\mathrm{S}_{\mathrm{ABC}} \quad \mathrm{S}_{\mathrm{GMRP}}=\mathrm{S}_{\mathrm{AQP}}-\mathrm{S}_{\mathrm{CQR}}-\mathrm{S}_{\mathrm{AHG}} \mathrm{S}_{\mathrm{CMH}} \\
& A_{(i, j)}=S_{(i, j)}-S_{(i-1, j)}-S_{(i, j+1)}+S_{(i-1, j+1)}
\end{aligned}
$$

(Eq. 2)

When $i<j, A_{(i, j)}=0$ 
Following the above equations, all $A_{(i, j)}$ can be precisely calculated.

Finally, $\mathrm{N}_{\mathrm{j}}$, the number of events detected in the $\mathrm{j}^{\text {th }}$ column can be calculated with the following equation:

$$
\mathrm{N}_{j}=2 *\left\{\sum_{i=j}^{n} \rho_{i} A_{(i, j)}+\left[h \Delta r-\sum_{i=j}^{n} A_{(i, j)}\right] \rho\right\}
$$

(Eq. 4)

As soon as $\mathrm{N}_{\mathrm{j}}$ and $A_{(i, j)}$ are known, $\rho_{i}$ will be calculated. 


\section{Supporting Information}

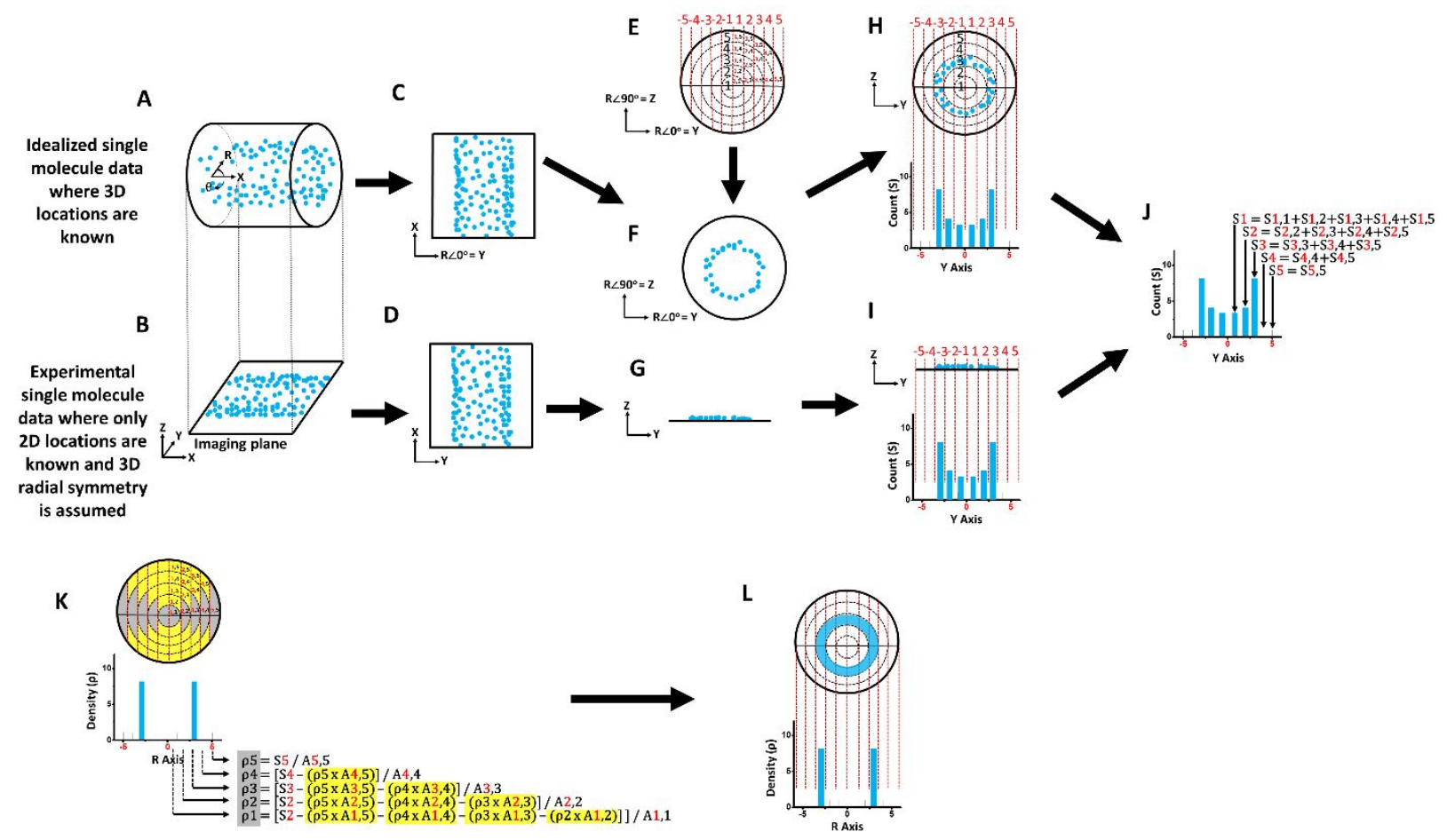

Figure S1. Further demonstration of 2D to 3D transformation process illustrating consistency of $y$ dimensional information regardless of $\mathrm{z}$ dimensional knowledge. (A) Idealized simulated single molecule data in $\mathrm{x}, \mathrm{y}$, and $\mathrm{z}$ dimensions. (B) Single molecule data from (A) projected to just the $\mathrm{x}$ and $\mathrm{y}$ dimensions, as it would be during the microscopy imaging process. (C) and (D) In the $\mathrm{x}$ and $\mathrm{y}$ dimensions, both the 3D single molecule data and the 2D projected data are identical. (E) An area matrix subdivided radially and axially to account for the radially symmetric structure from which the single molecule data originated. (F) Single molecule data from (A) viewed in the y and z dimensions. (G) Single molecule data from (B) viewed in the $\mathrm{y}$ and $\mathrm{z}$ dimensions. Since the data is projected, the $\mathrm{z}$ dimension is uniform. (H) and (I) y dimensional histograms from (F) and (G) are 
identical due to the fact that the $\mathrm{z}$ dimension is projected during the histogram process. (J) Each column in the y dimensional histogram in both (H) and (I) originate from the sum of the corresponding axial bins in the area matrix. (K) The 3D density histogram is calculated by using the outermost radial bin as a reference, calculating its density, then subtracting its contribution from the inner axial bins. The process is then repeated for the next most outer radial bin until the inner most radial bin is reached. (L) The final 3D density map gives a map of single molecule radial density rather than information about the 3D location of each single molecule. 


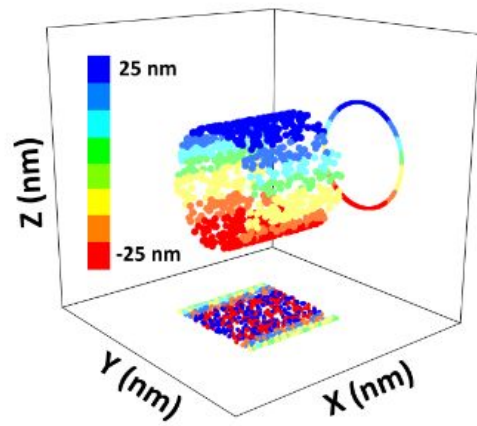

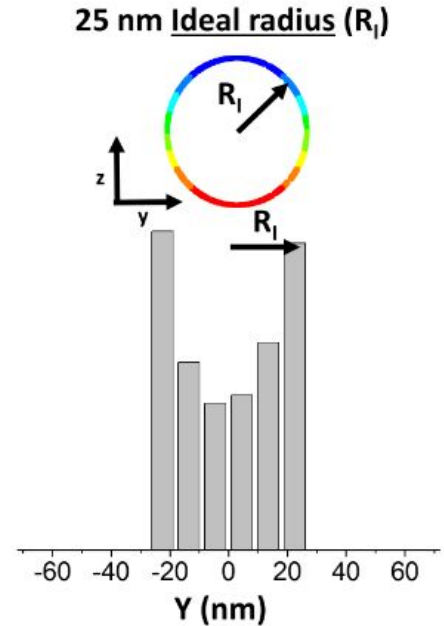

$10 \mathrm{~nm}$ localization error $\left(\sigma_{\mathrm{LE}}\right)$

C

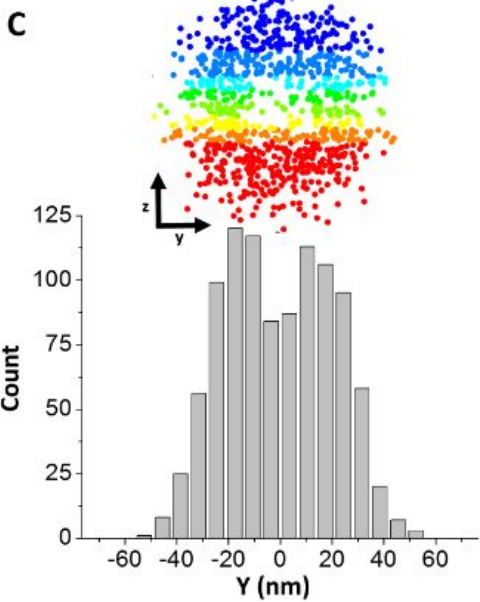

D

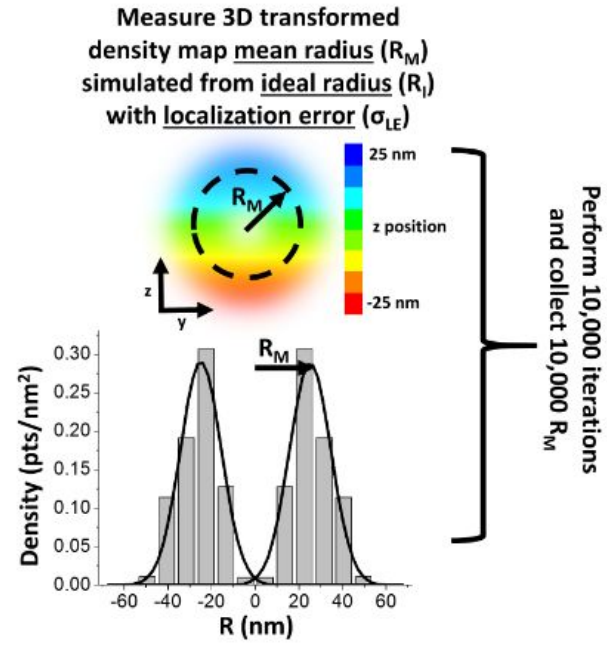

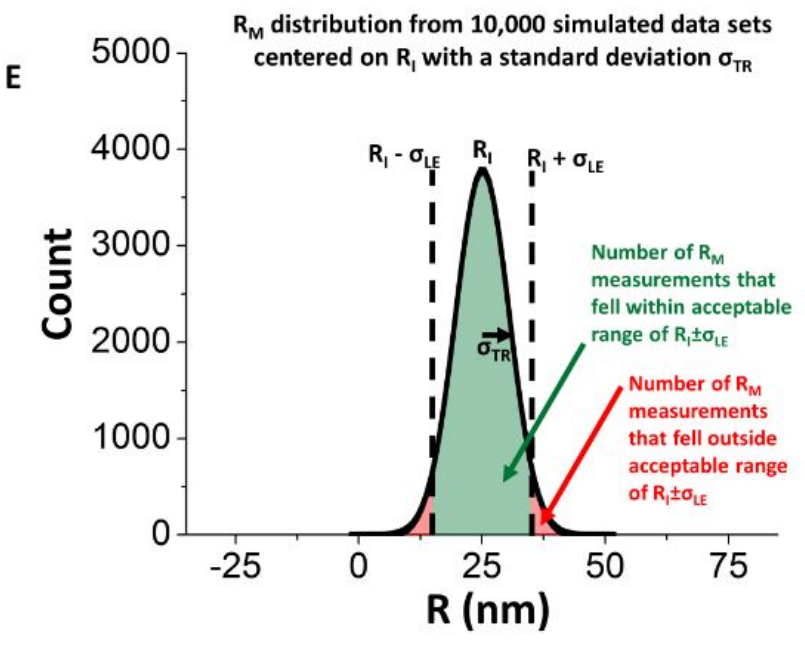

\section{$\mathbf{F}$}

Reproducibility $\%=\frac{\# \text { iterations within acceptable range }}{\text { Total } \# \text { of iterations }} \times 100 \%$

Figure S2. SPEED microscopy and 3D transformation reproducibility percentage using a simulation-based approach. For any given set of simulated data, the bin size is varied from $1 \mathrm{~nm}$ to the precision that is $10 \mathrm{~nm}$ in this example. (A) Data sets were simulated in three dimensions. Color bar indicates z position of the simulated points. (B) Each data set was simulated first with an ideal 25-nm radius (RI). (C) Subsequently, a localization error $\left(\sigma_{\mathrm{LE}}\right)$ of $10 \mathrm{~nm}$ was added to each point. (C) Using a 5-nm bin size for 
demonstration, the $2 \mathrm{D}$ histogram of the simulated data set with a $25-\mathrm{nm}$ radius and $10-\mathrm{nm}$ localization precision was determined. (D) 10,000 data sets were simulated with an ideal $25-\mathrm{nm}$ radius $\left(\mathrm{R}_{\mathrm{I}}\right)$ and a localization error $\left(\sigma_{\mathrm{LE}}\right)$ of $10 \mathrm{~nm}$. The resultant $3 \mathrm{D}$ histograms were then each fitted with a Gaussian function to localize the mean position of each peak, which is designated as the mean radius $R_{M}$. (E) The histogram for all the $R_{M}$ values was determined and the number of simulated data sets that fell within the acceptable range of $\mathrm{R}_{\mathrm{I}}^{ \pm \sigma_{\mathrm{LE}}}$ were counted. The acceptable range of $\mathrm{R}_{\mathrm{I}}^{ \pm} \sigma_{\mathrm{LE}}$ was chosen because, in principle, the Rayleigh criterion limited the resolution of any single 3D histogram to the spread of that distribution, which was due to the simulated localization error $\left(\sigma_{\mathrm{LE}}\right)$. After 10,000 simulations, the histogram for $R_{M}$ values converges on the mean $\left(R_{I}\right)$ from which they were originally sampled, while the spread of the $R_{M}$ histogram $\left(\sigma_{T R}\right)$ converges on a value that is due to the number of simulated points in each distribution and simulated localization error. (F) Reproducibility percentage was defined as the number of $R_{M}$ values that fell within the acceptable range of $\mathrm{R}_{\mathrm{I}}^{ \pm \sigma_{\mathrm{LE}}}$ divided by the total number of simulated data sets and multiplied by $100 \%$. 

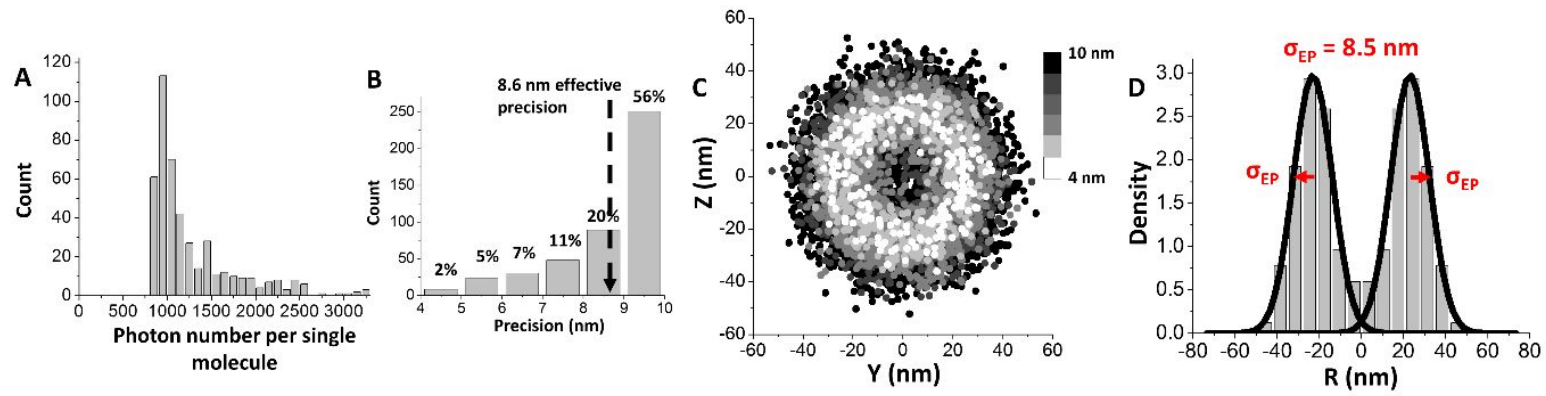

Figure S3. Minimum amount of experimental data points needed for a reliable 3D distribution of Imp $\beta 1$ in the NPC. (A) During the data selection process, single molecules with less than $\sim 800-1000$ photons are removed before the final $3 \mathrm{D}$ transformation analysis. (B) In the precision distribution, this corresponds to a cutoff precision of $10 \mathrm{~nm}$. Each whole number precision value below $10 \mathrm{~nm}$ threshold contributes a certain percentage to the overall weighted average precision of $8.6 \mathrm{~nm}$. (C) As an example, 10,000 single molecule points were simulated on an ideal radius $\left(R_{I}\right)$ of $25 \mathrm{~nm}$ and given a localization error based on the precision distribution from (A). Grayscale values indicate precision assigned to each single molecule. (D) The 3D transformation from (C) shows that the spread of each peak is approximately equal to the weighted average or "effective" precision $\left(\sigma_{\mathrm{EP}}\right)$ of the distribution rather than the cutoff precision. 
A
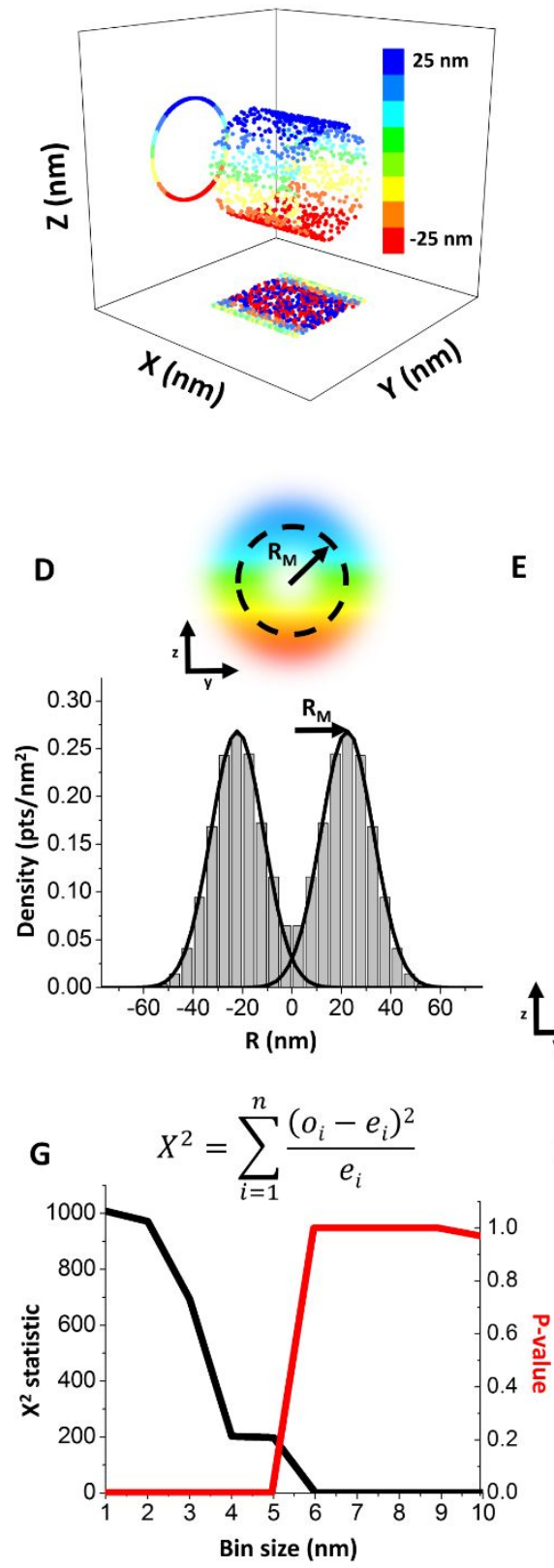

B
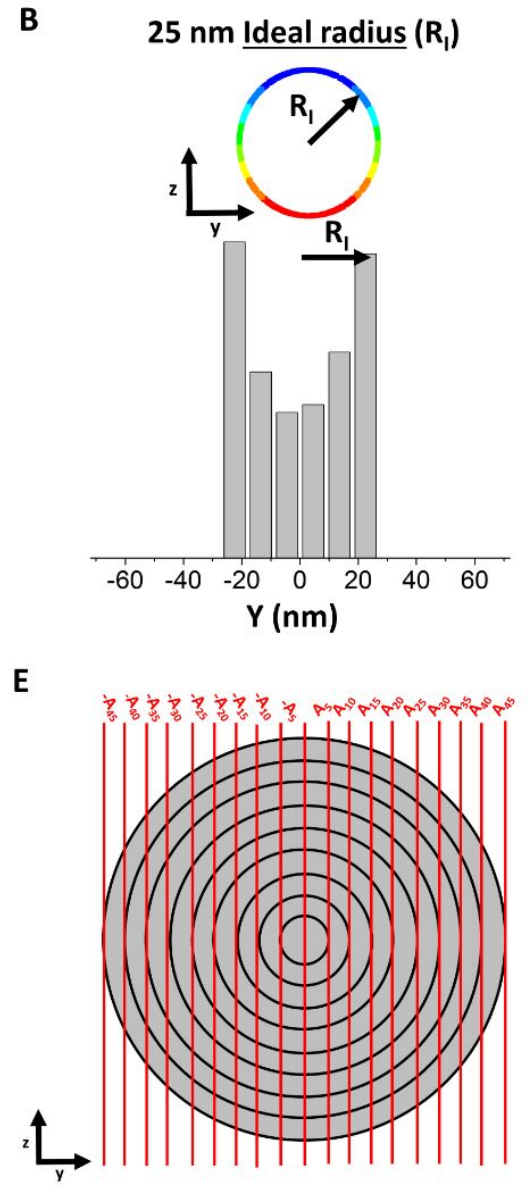

$10 \mathrm{~nm}$ localization error $\left(\sigma_{\mathrm{LE}}\right)$

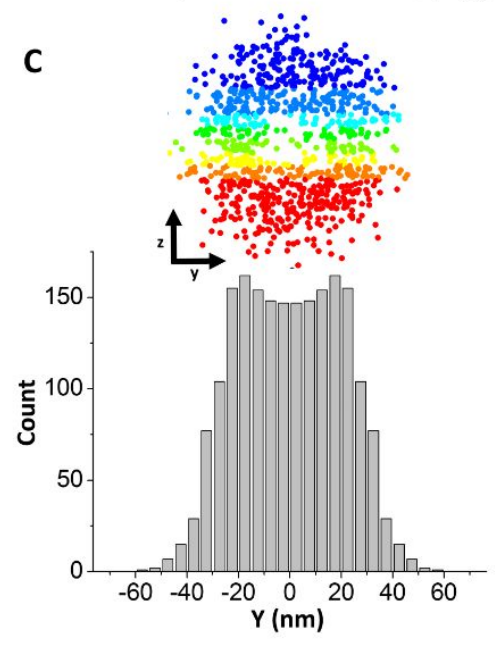

$\mathbf{F}$
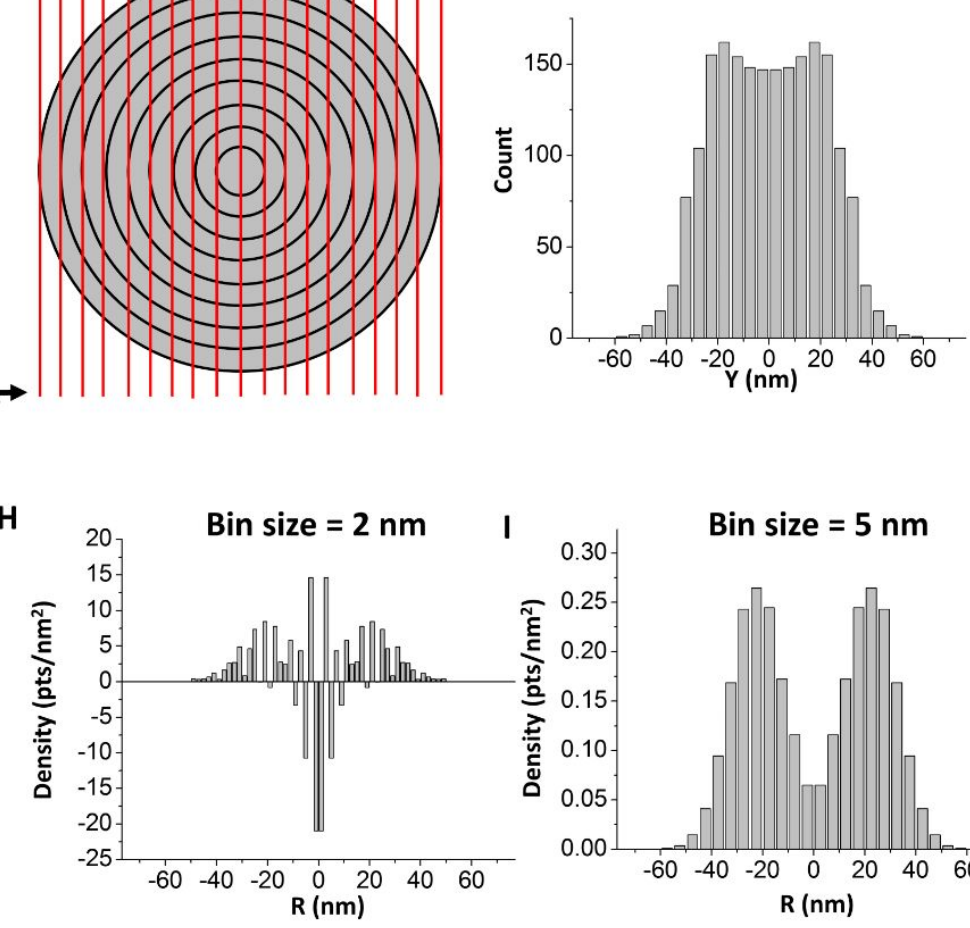

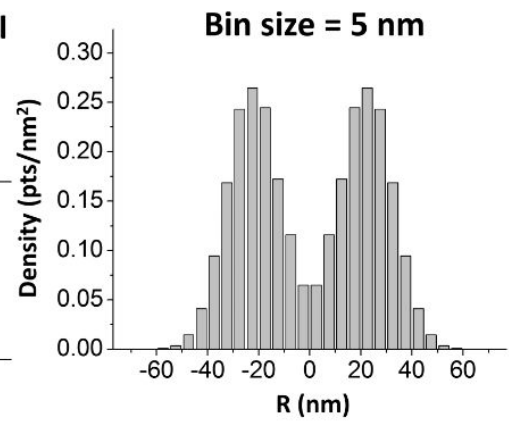

Figure S4. Optimal bin size determination using Chi-square error analysis. For any given set of simulated data, the bin size is varied from $1 \mathrm{~nm}$ to the precision that is $10 \mathrm{~nm}$ in this example. (A) Data sets were simulated in three dimensions. Color bar indicates z position of the simulated points. This representative data set contains 1,000 single molecule 
locations. (B) Each data sets was simulated first with an ideal 25-nm radius $\left(\mathrm{R}_{\mathrm{I}}\right)$. (C) Subsequently, a localization error $\left(\sigma_{\mathrm{LE}}\right)$ of $10 \mathrm{~nm}$ was added to each point. Using a 5-nm bin size for demonstration, the 2D histogram of the simulated data set with a $25-\mathrm{nm}$ radius and 10-nm localization precision was determined. (D) The 3D density histogram was then obtained via the 2D to 3D transformation algorithm and the peaks were fit with Gaussian distributions. (E) The 5-nm bin-size area matrix was calculated and multiplied by the 3D density distribution in (D) to reconstruct the 2D distribution (any negative values in the density distribution were set to zero) as shown in F. (F) The values of the reconstructed 2D distribution were then compared bin-by-bin to the original 2D distribution (as shown in C) using the Chi-square analysis equation where 'o' refers to the observed histogram values in $(\mathrm{F})$, 'e' refers to the expected histogram values in (C), ' $\mathrm{i}$ ' refers to the bin, and ' $\mathrm{n}$ ' refers to the total number of bins with histogram values in them. (G) The Chi-square test statistic and $\mathrm{p}$-value were then plotted across the potential bin size values. A p-value $\leq 0.01$ indicates that the 2D histograms in (C) and (F) are different from each other, suggesting the lack of enough data to allow sufficient sampling from each bin. On the other hand, a pvalue $>0.99$ suggests that the $2 \mathrm{D}$ histograms are statistically similar and likely have enough data points to accurately measure the value in each bin. Chi-square analysis was performed 1,000 times for each set of simulation parameters. (H) A 3D density histogram displaying large errors (negative density values) due to a bin size selection that was too small. Such errors occur when the single molecule density is not uniform throughout a given radial bin. This non-uniformity is due to under-sampling. (I) A 3D density histogram displaying no errors and a clear Gaussian distribution due to a properly selected bin size. A larger bin size can smooth the effects of under-sampling to a certain degree. 


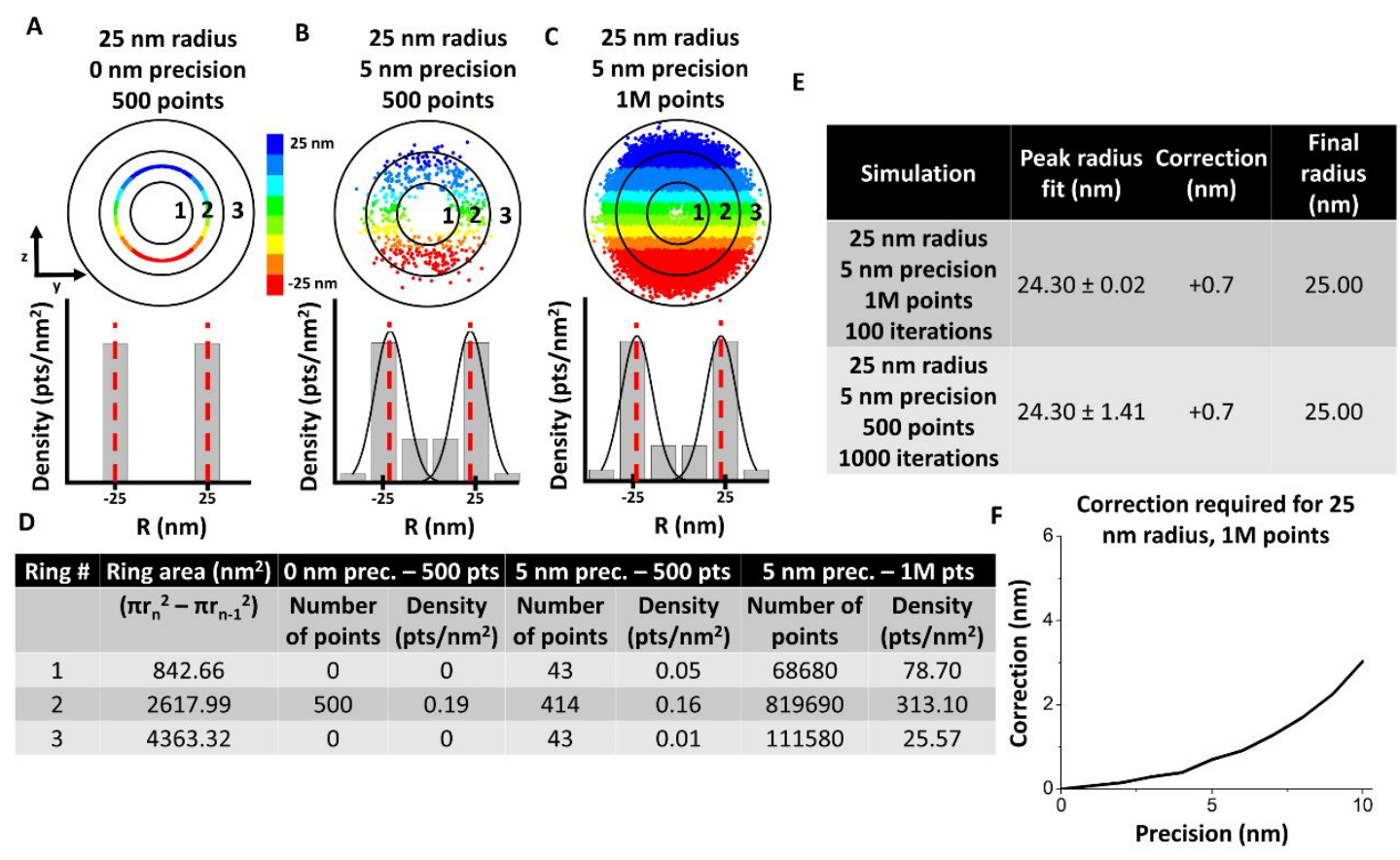

Figure S5. Sensitivity of inner bins necessitates slight correction of peak position during simulation. (A), (B), and (C) Simulated single molecule data and corresponding 3D density histogram for simulation with $25 \mathrm{~nm}$ radius, $0 \mathrm{~nm}$ localization error, and 500 points. Red dashed lines indicate mean peak fitting. (B) Simulated single molecule data and corresponding 3D density histogram for simulation with $25 \mathrm{~nm}$ radius, $5 \mathrm{~nm}$ localization error, and 500 points. Red dashed lines indicate mean peak fitting. (C) Simulated single molecule data and corresponding 3D density histogram for simulation with $25 \mathrm{~nm}$ radius, $5 \mathrm{~nm}$ localization error, and 1 million points. Red dashed lines indicate mean peak fitting. (D) Table showing the calculation to obtain each bin of the 3D density histogram. (E) Table showing that even one million points does reconstruct a precise 25 $\mathrm{nm}$ peak fitting due to the fact that the inner radial bins have smaller area and are slightly more sensitive to changes in density. (F) Correction required for each precision up to 10 
$\mathrm{nm}$ for a $25 \mathrm{~nm}$ radius. This correction process was performed before each simulation to accurately localize the $\mathrm{R}_{\mathrm{M}}$ density peak and correlate it to the ideal $\mathrm{R}_{\mathrm{I}}$ from which the data was simulated. 


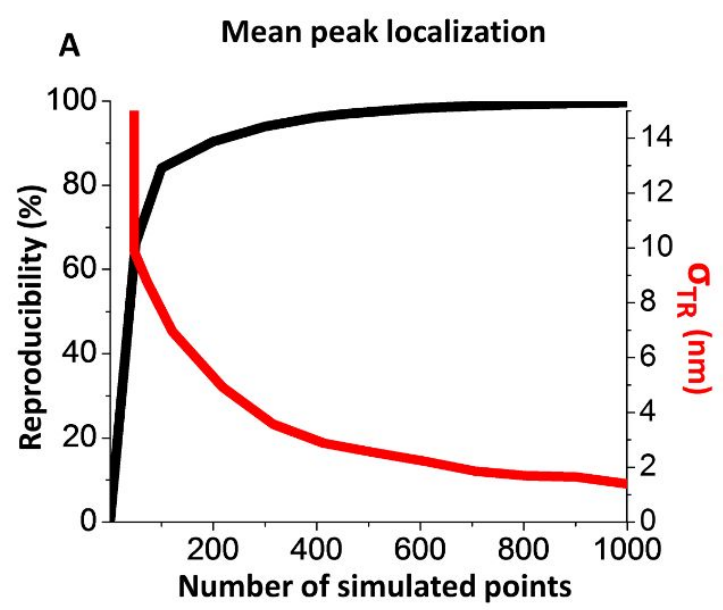

B
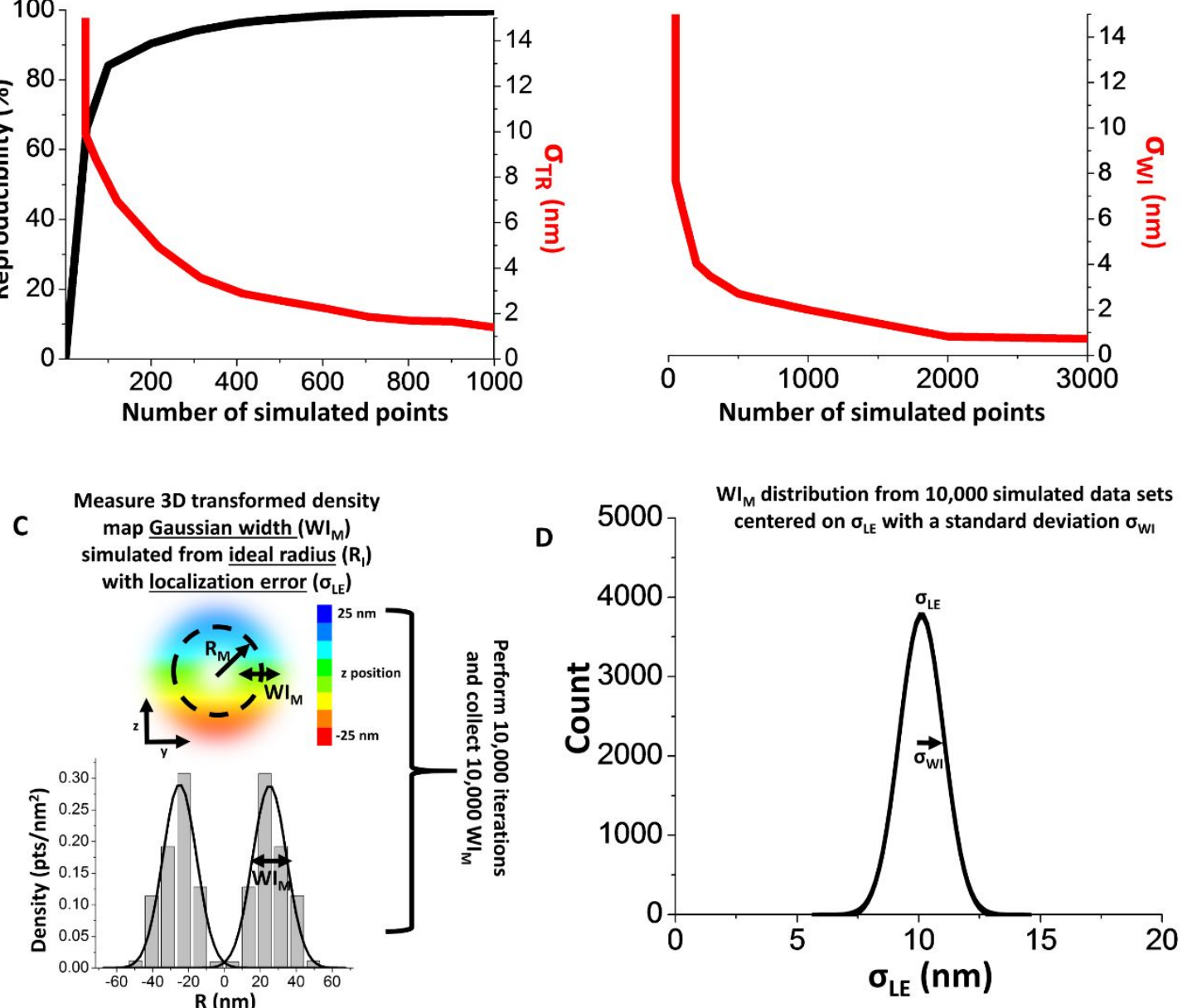

Figure S6. The accuracy of other parameters of the 3D density probability

distribution may be estimated as well. A) Plot of reproducibility percentage across

various simulated point numbers for the mean peak localization from Figure 4I

superimposed on the plot of $\sigma_{\mathrm{TR}}$, the corresponding nanometer value of mean $3 \mathrm{D}$ density peak localization error from which the reproducibility percentage was derived. B) Plot of

$\sigma_{\mathrm{WI}}$, the nanometer value of the mean 3D density peak Gaussian width. C) and D) The simulation was performed by the same method as Figure 4I and Figure S6A with the exception that the standard deviation of the Gaussian widths $\left(\sigma_{\mathrm{WI}}\right)$ was retained for analysis. 


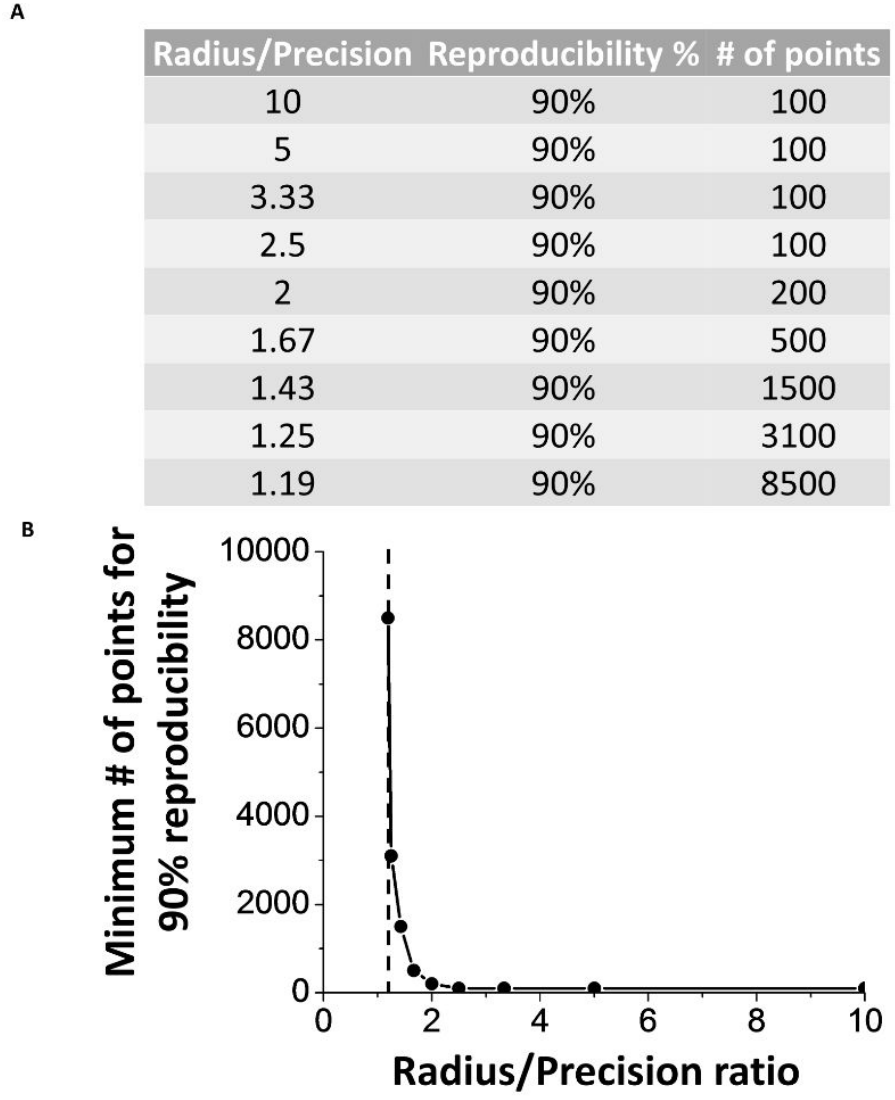

Figure $S 7$. The minimum number of points required to resolve a transport route above the radius/precision threshold. (A) Table illustrating the minimum point number requirements for $90 \%$ reproducibility across a range of radius/s.d. ratios which covers the experimental data in Figure 4. Point numbers were rounded to the nearest 100 points. \% route precision was calculated by dividing the transport route localization error $\left(\sigma_{\mathrm{TR}}\right)$ by the ideal radius $\left(\mathrm{R}_{\mathrm{I}}\right)$. (B) Graph showing the table from (A) plotted in black dots and solid lines down to the minimum distinguishable $\mathrm{R} / \mathrm{P}$ ratio threshold of 1.19 (dashed line). The green region of the graph represents the $\mathrm{R} / \mathrm{P}$ ratio range that can distinguish a transport route with an empty center (bimodal distribution in 3D density map; Figure 2) from a transport route with a filled center (single central peak in 3D density map; Figure 2). 
Colored dots represent the location of R/P ratio from experimental data from Figure 4. In the case of importin $\beta 1(\mathrm{R} / \mathrm{P}=2.3-3.65 ; 450$ points $)$, tubulin $(\mathrm{R} / \mathrm{P}=5.09 ; 112$ points $)$, and SSTR3 (R/P=7.34-12.7; 260 points), excess points above the threshold (100 points) were collected to ensure accurate localization of the transport routes. In the case of the GNC (3000 points), single molecule localization precision $\leq 5 \mathrm{~nm}$, an $\mathrm{R} / \mathrm{P}$ ratio of 0 , and the hollow structure of the GNC suggest that the accurate interpretation of the data are that it likely has a single central transport route. 